\title{
Novel Framework for Proposed Intelligent Examination System
}

\author{
Abdulrahman Abdullah Alghamdi, Mohammed Ateeq Alanezi and Z. Faizal Khan* \\ College of Computing and Information Technology, Shaqra University, Kingdom of Saudi Arabia; \\ alghamdia@su.edu.sa, alanezi.mohd@su.edu.sa, faizalkhan@su.edu.sa
}

\begin{abstract}
Objectives: Developing a computer-based system for examinations is the substitute for the current examination system based on paper. In recent days, the E-learning has become more popular because of its adaptability, integrity and user friendliness. In terms of the paper-based examinations, major challenge is the proctoring techniques used. In this paper, a novel method to avoid the presence of a proctor throughout the examination is proposed by an intelligencebasedexamination system. Methods: The system is designed with different complexity levels among the questions and also it acts as a tool for assessing the understanding of a student from the teaching materials. This method is proposed to improve the E-learning using intelligent question banks and examination system. Findings: This system can be timesaving and more efficient with an adequate level of security. The proposed methodology can be classified into two main phases such as the design of question bank along with its database, the design of the Artificial Intelligence based system for examination and its evaluation in order to improve the e-learning. Applications/Improvements: Future works in this system can be improved with the addition of theory-based questions and the integration of biometric based systems for enhancing the level of security.
\end{abstract}

Keywords: Artificial Intelligence, E-learning, Information Systems, Intelligent Question Bank System, Software Engineering

\section{Introduction}

Nowadays, the E-learning techniques ${ }^{-\frac{1-5}{5}}$ have rapidly developed and there are many types of E-learning based methodologies such as the traditional-based, online-based and the workplace etc. as well for education, through which everyone can perform the examinations. E-learning based on Information Technology (IT) has become the necessary resource from which an instructor needs to handle the examination. The instructor is the one who can develop the students by improving the skill of searching for resources, which then can be applied to their day to day activities. E-learning is a part of these activities. It is the easiest method of accessing the teaching materials especially for the students. It is also a method for connecting the instructors and the students. By this E-learning based technology, the students can handle their homework using the internet. The instructor can conduct online based examinations, can evaluate and revert back the students with their marks.

In this day and age, varieties of assessment and evaluation mechanisms are ${ }^{6,11}$ there in the education system. In general, majority of the instructors are conducting examinations based on the aptitude type of questions such as True-false type questions, Matching type of questions, Multiple Choice type questions or short answer type etc. For this process, each instructor needs to create the exam question paper manually, conduct the examination manually and then evaluate the answers manually. This manual process is tedious and it takes more time and effort to complete the examination and its evaluation process. Moreover, there may be a possibility of occurring errors during this evaluation. Hence, the need of an automatized system for conducting and evaluating examinations is necessary.

*Author for correspondence 
The intelligent algorithm will choose the already prepared test items, which were chosen in advance for creating the question bank system in order to be the repository for examination. Some test items will be collected and then will be chosen to be in the text based on the current test results. Furthermore, this system will use it's already obtained score in order to access the ability of understanding of the student as well as to improve the examination. The question bank system can reduce the duration to produce test items. As a result, it is an advantage for education: For example, there are some good test items, which enhance their standard and reliability. Artificial Intelligence $\frac{10}{10}$ will separate test items in many categories according to their difficulty. Moreover, it can choose test items to create exams according to learning objectives.

\section{Literature Survey}

Artificial Intelligence is the process of imitating human wisdom in computer, in many tasks such as ability, logical reasoning, thinking, recognition etc. This methodology makes the computer to do as humans perform ${ }^{4,5}$. In this case, the computer can be the expert because it is trained and tested by the experts. This technology will solve this problem effectively. Machine learning the process of training a computer to do the process done by humans learning using analgorithm. This algorithm can be supervised or unsupervised. The machine learning algorithm is trained to predict the result or to continue the process ${ }^{6,7}$. The computer can acknowledge the actual situation in order to decide effectively. Prediction of the result will be better according to the new data. Learning algorithms can be divided (according to learning processes) into four types: Supervised learning, unsupervised learning, semi-supervised learning and reinforcement learning.

$\operatorname{In}^{8}$ developed a novel adaptive based assessment methodology to process examinations. Their methodology improved the quality of examination system by creating an examination pattern based on the E-learning based system. In their system, they performed pre-assessment of the questions so the examination can be moderate as well as hard for the students. Depending on the pattern of the examination, answers are also prepared. Researchers' $\mathrm{in}^{9}$ proposed an assessment system which is adaptive in type with the combination of an examination database. In their approach, the efficiency of the examination can be increased by choosing the questions in series of either asking a multiple type. This computer-assisted examination system reduced the work of creating examination as well as the proctoring.

Multiple assessments with formulas are proposed by 11 - in order to create serial examinations and also for evaluating continuously. Their method is based ona multiple criteria. Their online based examination system can be used in the bank system. It can be gradually changed dynamically for other examination systems for the students' learning purposes.

\section{Proposed Methodology}

The proposed intelligent examination system consists of four modules such as the user management, course management, question and its answer management, examination and its evaluation management etc. Its architecture is shown in Figure 1. The proposed system is designed by using machine learning based techniques. It also applied logical design to get the suitable intelligent question bank system framework.

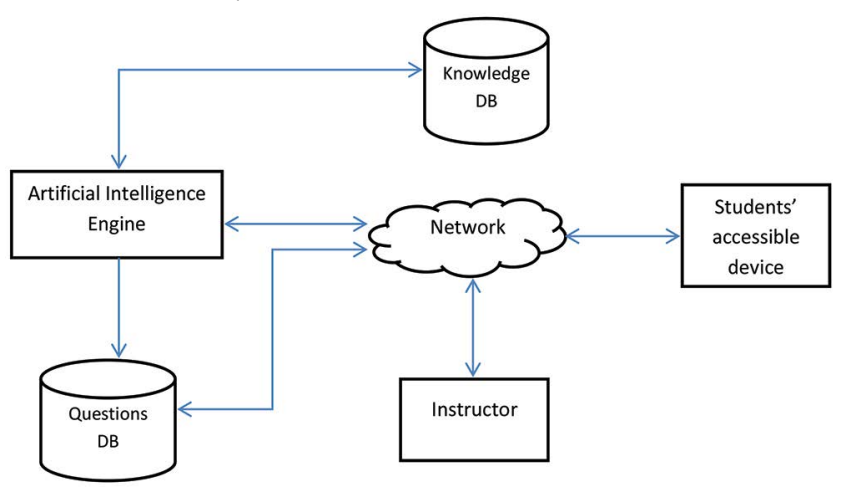

Figure 1. Architecture of the proposed framework.

\subsection{Question Bank System}

In this method, the question bank system consists of sets of 100 questions which are created from to the students' syllabuses which is a mixture of medium, easy and hard. Selection of the questions is automatized and intelligencebased. The passing percentage of each level of exam is 70 . Depending up on the level of marks obtained in each level and in each question, the algorithm will choose 
the level of questions. The evaluation process is also automatized so that the algorithm can find the level of understanding of students in each section of the subject. The main advantage of the proposed method is analysing the student's understanding level and mastery level in the respective chapters. The proposed methodology will adjust the questions based the students understanding level and mastery level.

The question database will have the questions uploaded by instructors. Each question has the following features:

- Aptitude type.

- Question answers with relevancy to the correct answer for instance; correct answer will have 100\% relevant, wrong answers would have 75\% relevant, 50\% relevant, $25 \%$ relevant and $0 \%$ relevant.

- Question category/domain.

- Question level: As medium, easy and hard.

\subsection{Evaluation System}

In this section, an Artificial Intelligence based intelligent system is used for allocating the marks for each questions based on the correct answers. The algorithm is trained with questions and its respective answers. The AI agent is an intelligent module which allocates the correct answers for the appropriate questions and it also allocates the set of questions to a specific student based on the register number so that the question sets cannot be repeated among the others. The AI based subsystem can generate the questions and send them to the device where the student can access the question and he can write the answers.

- AI agent: Artificial Intelligence (AI) based agent is an intelligent module which has the following tasks:

- Generate questions to be sent to students.

- Generate a knowledge base from students' answers, with feedback to know more about some explanations of students' answers and areas of weakness and areas of strength.

- Student Accessible Device: Access the questions, answer them and submit the answers.

- Instructor: Instructor can do the following tasks:
- Add/Modify/Delete Question.

- Generate exam with a predefined number of questions and the percentage of each level of questions.

- Knowledge DB: Stores the generated knowledge from student answers feedback via AI engine.

\section{Modules of the Proposed System}

The proposed system of an intelligent question bank and examination system consists of four parts : 1 . User Management; 2. Course Management; 3. Question and its answer Management; 4. Examination and its evaluation Management.

\subsection{User Management}

The role of user management is to monitor the functions such as login, editing the profile and allowing permission for the users for accessing the system. Login function monitors the users who were accessing the system. It also maintains the correct username, password and sign up function before login to the system. Also, the Users can edit their profiles, by changing the password, email and their profile picture etc. It manages the setting permission to different types of users such as the student, teacher or administrator.

\subsection{Course Management}

The role of user management is to monitor the course Management by add and delete the courses. Setting the courses by Set items, such as grouping courses, evaluation rate calculation etc. and AI for grouping the courses automatically based on the register numbers of the student.

\subsection{Question and its Answer Management}

Question and its answer management is to monitor the questions and its corresponding answers by adding, deleting and updating the questions and its corresponding answers., setting the questions, assigning the answers. Artificial Intelligence based methodology for grouping 
the questions and its answers automatically based on the register numbers of the students.

\subsection{Examination and its Evaluation Management}

Setting Examination, Monitoring Examination, Evaluation of Examination, Declaration of the results and suggestions and an Artificial Intelligence based methodology are the tasks done by the Examination and its Evaluation Management system. Setting the examination comprises of setting the date, time for the starting and finishing the exam etc. Monitoring examination consists of checking the students who are performing or finished the exam. Declaration of the results and suggestions comprises of evaluating the examination, writing reports based on the results obtained. Applying AI based algorithmfor choosing theoverall examination and its evaluation management is also to be done.

\section{Conclusion and Future Enhancements}

In this study, an intelligent computer-based question bank and an examination system is proposed. This consists of four modules, which are the user management, course management, question and its answer management and the examination and its evaluation Management. An AI based algorithm is used for performing all the modules present in the proposed system. The proposed methodology is made intelligent using the AI based methods. This method is proposed to improve the E-learning using an intelligent question bank and examination system. The system is designed with different complexity levels among the questions and also it acts as a tool for assessing the understanding of student from the teaching materials. The AI bases algorithm is applied to all the four modules of the proposed system. Future works in this system can be done with the inclusion of theory-based questions and the integration of additional security measures such as the biometric based systems.

\section{Acknowledgement}

We would like to thank the Shaqra University to support this research. This work had been financed under Shaqra University, grant Nr. D180000/G01/N055.

\section{References}

1. Whitehouse C. Item banks and on-demand tests. The Assessment and Qualifications Alliance (AQA), Centre for Education Research and Policy; 2012. p. 1-4.

2. Stucky BD, Edelen MO, Sherbourne CD. Developing an item bank and short forms that assess the impact of asthma on quality of life. Respiratory Medicine. 2014; 108(2):252-63. PMid: 24411842 PMCid: PMC4065171. https://doi. org/10.1016/j.rmed.2013.12.008

3. Banerjee S, Rao NJ, Ramanathan C. Designing item banks in alignment with course outcomes for engineering courses. Proc IEEE 8th International Conference on Technology for Education; 2016. https://doi.org/10.1109/T4E.2016.039

4. Akrimi JA, Ahmad AR, George LE, Aziz S. Review of Artificial Intelligence. International Journal of Science and Research. 2013; 2(2):487-506.

5. Hammond K. Practical Artificial Intelligence for dummies. 1st ed. Narrative Science. 2015. p. 1-30.

6. Portugal I, Alencar P, Cowan D. The use of machine learning algorithms in recommender systems: A Systematic Review. Expert Systems with Applications. 2018; 97:205-27.

7. Kalyankar GD, Poojara SR, Dharwadkar NV. Predictive analysis of diabetic patient data using machine learning and hadoop. Proc 2017 International Conference on I-SMAC (IoT in Social, Mobile, Analytics and Cloud); 2017. p. 61924. https://doi.org/10.1109/I-SMAC.2017.8058253

8. Thirapunmetee P, Piriyasurawong P. Development of item bank framework on cloud computing system. Academic and Research Journal. Rajamangala University of Technology PhraNakhon Science and Technology; 2014; 8(2).

9. Wang M, Wang C, Lee C, Lin S, Hung P. Type-2 Fuzzy set construction and application for adaptive student assessment system. Proc 2014 IEEE International Conference on Fuzzy Systems; 2014. p. 888-94. https://doi. org/10.1109/FUZZ-IEEE.2014.6891894

10. Khater E, Hegazy A, Shehab ME. Ontology-based adaptive examination system in E-learning management systems. Proc 2015 IEEE 7th International Conference on Intelligent Computing and Information Systems; 2015. p. 243-50. https://doi.org/10.1109/IntelCIS.2015.7397228

11. Cong Y. Functional design of English online examination system based on ASP technology. Proc 3rd International Conference on Education, Management, Arts, Economics and Social Science; 2016. p. 1140-4. 